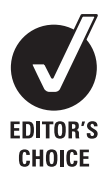

- An additional appendix is published online only at http:// adc.bmj.com/content/vol94/ issue 5

${ }^{1}$ Neonatal Unit, Elizabeth Garrett Anderson \& Obstetric Hospitals, London, UK; ${ }^{2}$ NICU, Monash

Medical Centre, Melbourne, Australia; ${ }^{3}$ Centre for Paediatric Epidemiology and Biostatistics, University College London, London, UK

\section{Correspondence to:}

Janet M Rennie, Neonatal Unit, Elizabeth Garrett Anderson \& Obstetric Hospitals, University College London Hospitals, Euston Road, London NW1 2PO, UK; janet.rennie@uclh.org

Accepted 6 October 2008 Published Online First 10 November 2008

\title{
Range of UK practice regarding thresholds for phototherapy and exchange transfusion in neonatal hyperbilirubinaemia
}

\author{
J M Rennie, ${ }^{1}$ A Sehgal, ${ }^{2}$ A De, ${ }^{1}$ G S Kendall, ${ }^{1}$ T J Cole ${ }^{3}$
}

\begin{abstract}
Objective: To establish the range of opinion regarding thresholds at which phototherapy and exchange transfusion are used to treat neonatal hyperbilirubinaemia in the UK.
\end{abstract}

Design: A survey of existing charts and guidelines collected from around the UK. Threshold levels were extracted from the charts and entered into an Excel spreadsheet. Filters were applied to analyse subsets of guidelines, and calculations were carried out to analyse the rate of rise of bilirubin (in $\mu \mathrm{mol} / / / \mathrm{h}$ ) between the origin and the plateau, where this was possible.

Results: Of 263 hospitals contacted, 163 submitted guidelines, of which most were in the form of individual charts. There was wide variation in the choice of the threshold levels at which treatment was recommended, particularly in preterm babies. At 28 weeks, for example, the range at which phototherapy was recommended extended from $100 \mu \mathrm{mol} / /$ to $250 \mu \mathrm{mol} / \mathrm{l}$, and the upper limit was even higher if data from units which used a single guideline for preterm babies of all gestations were included. There was variation in the choice of the origin of the graph and the time at which the plateau commenced (and hence the slope), whether "sickness" criteria should be adopted, and what those criteria should be. Many charts were confusing, poorly presented, sketchily drawn and lacked proper gridlines or axis labels.

Conclusions: For such a vitally important topic it is disappointing that there is little existing consensus and no national guidance in the UK. Guidelines for England, Wales and Northern Ireland will be available from 2010, when the National Institute for Health and Clinical Excellence completes its review.

Phototherapy represented an important advance in the treatment of jaundice, enabling the effective and relatively rapid reduction of high bilirubin levels and facilitating the prevention of kernicterus. Phototherapy is cheap, readily available, easy to administer and has few side effects. Given the devastating nature of kernicterus, any risk-balance analysis emerges strongly in favour of the liberal use of phototherapy. This is particularly the case in the preterm baby, who is already separated from his or her parents and whose fluid balance and temperature control are under close scrutiny. Exchange transfusion is the only alternative to phototherapy for controlling hyperbilirubinaemia. Exchange transfusion, while undoubtedly an effective treatment which was a milestone in the history of neonatal care, is an invasive intensive care procedure which carries a small risk of death

\section{What is already known on this topic}

- Different hospitals use different charts and guidelines to determine thresholds for use of phototherapy and exchange transfusion.

\section{What this study adds}

- Quantification of the wide variation in opinion and practice regarding threshold values for the use of phototherapy and exchange transfusion in term and preterm babies in the UK, with proposals for a consensus guideline which could be subject to future modification on the basis of audit.

or a serious complication such as thrombosis, embolus, sepsis and necrotising enterocolitis. ${ }^{1}$

All neonatal services use phototherapy, and those who work in this service need clear guidance on how best to deploy this effective treatment. Because kernicterus is so rare, and because kernicterus is not simply a function of the total bilirubin level but involves an interaction between the level of free unconjugated bilirubin and the blood-brain barrier, this clear guidance is difficult to formulate. The rarity of kernicterus means that there never will be a robust evidence base for these essential guidelines, and this has hampered progress in this area. Decisions regarding the management of hyperbilirubinaemia have to be based on consensus, case reports and experience, while accepting that any such guideline will fail on occasion; kernicterus has occurred in healthy preterm babies at very low levels of bilirubin, ${ }^{2}{ }^{3}$ and there are anecdotal reports of kernicterus in babies with high levels of conjugated bilirubin. ${ }^{4}$

There is currently no national guideline in the UK; the American Academy of Pediatrics (AAP) has issued two "practice parameters" which have been widely studied in countries other than the USA. ${ }^{56}$ The AAP guideline does not include guidance for babies less than 35 weeks of gestation, although some helpful advice has been published in the Archives. $^{78}$ The AAP guideline was based on an extensive review of available evidence, ${ }^{9}$ and the recommendations included promotion of breast feeding, a systematic risk assessment, and estimation of transcutaneous or total serum bilirubin on 
all babies who were thought to be jaundiced in the first $24 \mathrm{~h}$ with a repeat measurement if the bilirubin level fell into a highrisk zone (defined using an hour-specific nomogram ${ }^{10}$ ).

In the UK, there have been many calls for a national guideline, which have at last been acknowledged. The National Institute for Health and Clinical Excellence (NICE) has started the process of developing a guideline for neonatal jaundice. Once this is available, if a register of cases of kernicterus can be developed, some progress could at last be made towards minimising the risk of kernicterus by regular reappraisal of the levels chosen against cases reported. In our view this "Delphi" approach is the only way forward in an area which has otherwise stagnated for over 30 years, and in which there are significant variations in current practice. With this aim in mind, we set out to survey current UK practice regarding treatment guidelines for hyperbilirubinaemia in term and preterm babies.

\section{METHODS}

\section{Survey}

We requested a copy of the local guideline for the management of jaundice from the lead clinician in each of the 263 neonatal units who are listed as providing neonatal intensive care in the UK. Stamped addressed envelopes were provided for the reply. The survey was carried out in the first months of 2005. An attempt was made to contact a different individual in units who did not respond but no attempt was made to analyse the nonresponding units in terms of level of unit or geographical location.

\section{Statistics}

Bilirubin levels were extracted from each of the graphical charts we received, and entered into an Excel spreadsheet. Each curve was summarised as a series of straight line segments that captured the shape of the curve, by recording the time (in decimal days) and corresponding bilirubin level at the start and end of each segment. Each point was flagged as being at the "start", "middle" or "end" of the curve, the "end" point indicating both the start time and level of the curve's plateau. Charts for babies of different weights and gestations were recorded separately, as were charts for phototherapy and exchange levels, together with the criteria used in the chart in question.

Filters were designed for use within Excel so that specific information could be easily extracted (for example, "end" point of the curve, phototherapy only, 33-36 weeks' gestation). Bilirubin levels for specific groups were extracted in this way and transferred into Prism version 4.03 (Graph Pad Software Inc, California, USA) for further statistical analysis and graphing.

\section{Ethics}

Ethical approval was not sought for this survey. No patient information was involved; no patient or individual neonatal unit can be identified and as such no approval was required.

\section{RESULTS}

Of the 263 hospitals contacted, 163 responded, of which 140 sent information which we could interpret.

Thirteen of these hospitals (9\%) were using formulae for deciding when to start phototherapy; two used a weight-based formula and the remainder the formula: bilirubin in $\mu \mathrm{mol} / \mathrm{l}=$ gestational age $\times 10-100$. Very few of the hospitals which sent formula-based guidelines stated what to do in the first $72 \mathrm{~h}$ of life, when bilirubin levels are still rising and treatment levels need to be lower. Two hospitals used a chart with gestation on the $\mathrm{x}$ axis rather than time, which also means that no guidance is provided before the bilirubin levels have reached a plateau. All other charts had time along the $\mathrm{x}$ axis, although some did not specify any units of time.

The charts we studied varied enormously in the level of detail of their content, and the quality of presentation. Many charts had been copied and recopied over the years and were almost indecipherable. Others had been sketched freehand originally and had no graphical gridlines at all. Many did not extend beyond $72 \mathrm{~h}$, making plotting at later ages difficult, and some lacked any units on the $\mathrm{x}$ axis. Some were neatly presented on coloured graph paper and laminated, but had no specific guidance for preterm babies, or else grouped all preterm babies together.

The most common chart we received was that originally designed by Rodney Rivers for use at St Mary's Hospital in London (unpublished). This was returned by 19 hospitals (13\%), mostly from around London. The St Mary's Manchester chart was in use by seven hospitals. Thirteen hospitals (9\%) are using the chart originally published by Finlay and Tucker, ${ }^{11}$ mostly with the Dodd modification for preterm babies. ${ }^{12}$ This chart does not distinguish subgroups of preterm babies. Nine hospitals $(6 \%)$ are using the original Cockington weight-based chart. $^{13}$

\section{The choice of the origin of the graph}

Some charts drew a line from the origin to the plateau of the graph, implying a cord blood bilirubin level of 0 . Two other charts started the line at a level of $225 \mu \mathrm{mol} / \mathrm{l}$ on the $\mathrm{y}$ axis at the time of birth. If these were excluded from the analysis the median value which was assumed to indicate a need for treatment based on cord blood levels was $50 \mu \mathrm{mol} / 1$ with an interquartile range of $30-70 \mu \mathrm{mol} / 1$. The published range for cord blood bilirubin levels is usually quoted as $20-35 \mu \mathrm{mol} / \mathrm{l}$, and a recent study from Germany found that an umbilical serum bilirubin level of $>30 \mu \mathrm{mol} / 1$ was associated with an increased risk of requiring phototherapy. ${ }^{14}$ Traditionally, a level of $80 \mu \mathrm{mol} / \mathrm{l}$ in cord blood has been considered an indication for exchange transfusion in Rhesus disease.

\section{The initial slope of the graph}

The huge variation in the choice of origin and plateau together with the variability in the start time of the plateau resulted in a very wide range of slopes for lines constructed over the first 48-72 h. The median value for the point of inflexion between the rising slope and the plateau was $72 \mathrm{~h}$; the 25 th percentile was $48 \mathrm{~h}$ and the 75 th percentile value was $86 \mathrm{~h}$. However, the range was very wide ( 6 h to 14 days), with several guidelines drawn to suggest a continuously rising slope even after 7 days of age.

This wide range of opinion in the position of the plateau phase inevitably produced a wide variation in the rate of rise which was considered to represent normal physiology during the first days of life. Analysis of 880 different slopes produced a median rate of rise during the first 3 days or so (see above) of $3.5 \mu \mathrm{mol} / \mathrm{l} / \mathrm{h}$. Again the range of opinion was wide, with the 25 th percentile at $2.08 \mu \mathrm{mol} / \mathrm{l} / \mathrm{h}$ and the $75 \mathrm{th}$ percentile at $4.66 \mu \mathrm{mol} / \mathrm{l} / \mathrm{h}$. The maximum rate of rise was plotted as $14.1 \mu \mathrm{mol} / \mathrm{l} / \mathrm{h}$, which most authorities would consider to represent a pathological rate of rise, suggesting haemolysis. Forty-six of the graphs analysed for rate of rise had a slope of $>10 \mu \mathrm{mol} / \mathrm{l} / \mathrm{h}$ before the plateau was reached. 
Figure 1 A. Histogram showing the number of hospitals choosing particular levels of bilirubin at which phototherapy is commenced in term babies. $B$. Histogram showing the number of hospitals choosing particular levels of bilirubin at which an exchange transfusion is recommended in term babies.

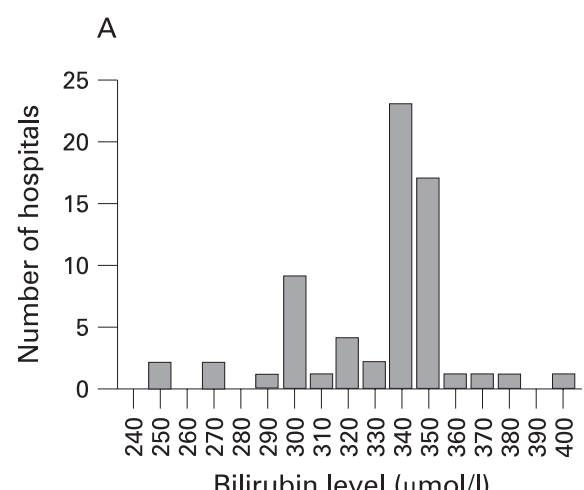

Bilirubin level $(\mu \mathrm{mol} / \mathrm{l})$

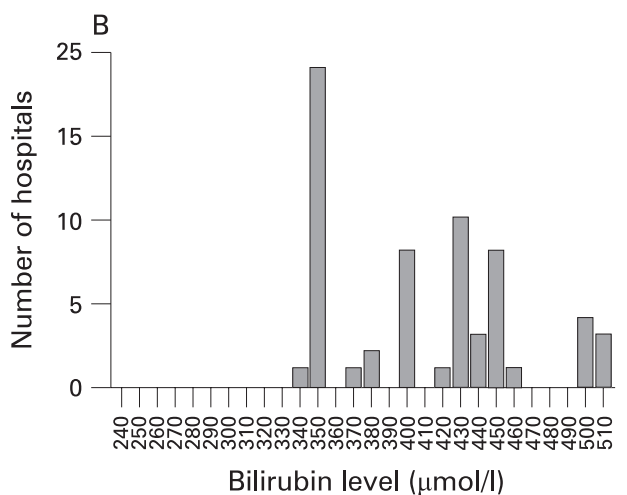

Recent studies using transcutaneous bilirubin measurements have shown that the rate of rise of bilirubin in the first $72 \mathrm{~h}$ is not linear, but the 95th percentile for the period between 6 and $24 \mathrm{~h}$ (at $>35$ weeks) was $3.74 \mu \mathrm{mol} / \mathrm{l} / \mathrm{h}{ }^{15}$

\section{Sickness criteria}

Fifty of the guidelines we studied suggested that levels should be modified in the presence of illness; the criteria and the degree to which they were specified varied. Some units specified haemolysis as a specific subgroup and provided separate charts; others used criteria such as acidosis or sepsis, many not further defined. The way in which haemolysis was recognised was not generally defined. Hansen reported 19 different "sickness" criteria ranging from hypoglycaemia to polycythaemia in his survey of international practice, and it is clear that UK guidelines are similarly variable in this respect. ${ }^{16}$

\section{The range of bilirubin levels chosen for action lines in term babies}

The range of opinion regarding the level of bilirubin at which phototherapy should be commenced in a healthy term baby was between 250 and $400 \mu \mathrm{mol} / 1$, with a median value of $340 \mu \mathrm{mol} / 1$ (fig 1A). For exchange transfusion, the range was between 340 and $510 \mu \mathrm{mol} / 1$ with a median value of $400 \mu \mathrm{mol} / \mathrm{l}$ (fig $1 \mathrm{~B}$ ). Twenty hospitals chose a value of $350 \mu \mathrm{mol} / \mathrm{l}$ for exchange transfusion in a healthy term baby. We did not collect information on how many exchange transfusions were actually performed at this level.

\section{The range of bilirubin levels chosen for action lines in preterm babies}

Plotting the raw data for the charts we analysed showed an enormous range of guidance regarding the use of phototherapy at different gestational ages. For example, at 26 weeks' gestation the recommendations varied from 80 to $330 \mu \mathrm{mol} / 1$ with a median of $150 \mu \mathrm{mol} / \mathrm{l}$ (table 1). Examination of the raw data shows that there is a cluster of guidelines with recommendations for phototherapy above $250 \mu \mathrm{mol} / \mathrm{l}$ at very low gestations, and these arise mainly from charts which have a single category of preterm babies rather than subgroups. ${ }^{12}$

We re-analysed these data excluding values $>250 \mu \mathrm{mol} / \mathrm{l}$ up to and including a gestational age of 32 weeks, and added the gestation-specific guidelines generated by the hospitals that use a formula. This produced a consensus chart which reflects a reasonable range of current UK practice, although there is still wide variation (fig 2).

\section{Guidance regarding handling direct and indirect bilirubin}

No guideline gave any advice regarding whether the direct component of total bilirubin should be subtracted from the total when making a decision on whether to perform an exchange transfusion. Individual practice varies considerably in this regard, and most of the major textbooks are virtually silent on the topic. ${ }^{17}$ The AAP guidelines recommend using total serum bilirubin for all management decisions except when the direct (conjugated) bilirubin level is around $50 \%$ of the total.

\section{DISCUSSION}

Our results of a UK-wide survey are similar to those of a smaller recent audit carried out in the North of England; $90 \%$ of units

Table 1 Range of serum bilirubin values (in $\mu \mathrm{mol} / \mathrm{l}$ ) used as threshold values for phototherapy, by each week of gestation between 23 and 36 weeks

\begin{tabular}{lrrrrrrrrrrrrrr}
\hline $\begin{array}{l}\text { Week of } \\
\text { gestation }\end{array}$ & 23 & 24 & 25 & 26 & 27 & 28 & 29 & 30 & 31 & 32 & 33 & 34 & 35 & 36 \\
$\begin{array}{l}\text { Number of } \\
\text { observations }\end{array}$ & 36 & 50 & 51 & 54 & 56 & 76 & 74 & 78 & 83 & 69 & 70 & 103 & 80 & 86 \\
Minimum & 100 & 80 & 80 & 80 & 80 & 80 & 80 & 100 & 100 & 150 & 150 & 150 & 200 & 200 \\
25th percentile & 120 & 90 & 100 & 100 & 115 & 100 & 150 & 150 & 160 & 200 & 200 & 220 & 250 & 250 \\
Median & 150 & 147 & 150 & 150 & 160 & 160 & 170 & 165 & 200 & 220 & 235 & 250 & 250 & 250 \\
75th percentile & 250 & 190 & 180 & 175 & 175 & 180 & 200 & 200 & 240 & 250 & 250 & 270 & 270 & 280 \\
Maximum & 330 & 330 & 330 & 330 & 330 & 330 & 330 & 330 & 330 & 330 & 33 & 370 & 370 & 380 \\
Mean & 181 & 155 & 155 & 155 & 163 & 156 & 175 & 176 & 198 & 224 & 226 & 240 & 262 & 269 \\
Standard & 69 & 72 & 72 & 70 & 67 & 63 & 55 & 53 & 45 & 45 & 45 & 43 & 35 & 39 \\
deviation & & & & & & & & & & & & 215 \\
Lower 95\% & 158 & 134 & 135 & 137 & 146 & 142 & 163 & 164 & 188 & 213 & 215 & 231 & 255 & 261 \\
Upper 95\% & 205 & 176 & 175 & 175 & 182 & 171 & 189 & 189 & 208 & 235 & 237 & 248 & 271 & 278 \\
\hline
\end{tabular}


Figure 2 Box and whiskers plot showing the median and range of bilirubin levels chosen as threshold levels for commencing phototherapy at each week of gestation after $72 \mathrm{~h}$ of life. Plot generated after excluding hospitals who had only one guideline for all preterm babies $<37$ weeks, and including data generated for hospitals which used a formula of: gestational age $\times 10-100$ to determine this threshold.

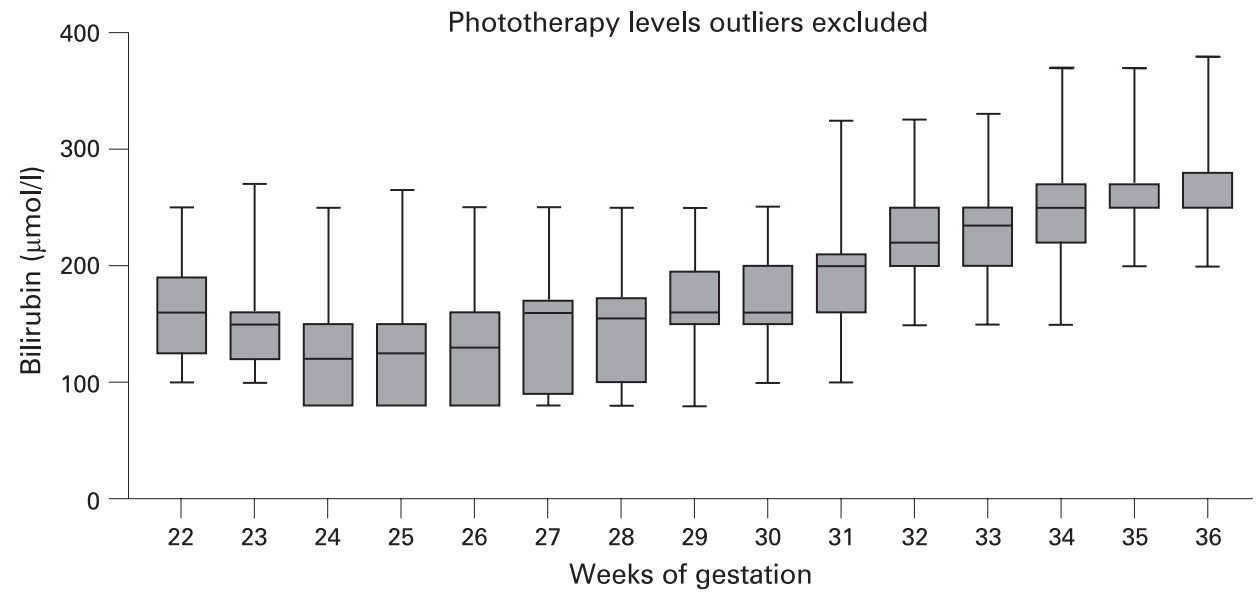

are using charts rather than formulae, most units do not know the derivation of their charts, and there is a wide variation in protocols. ${ }^{18}$ The quality of presentation and clarity of the advice offered was disappointing for such a common and important clinical problem. We agree with Skae and colleagues that there is an urgent need to develop standardised UK guidelines, and we hope that our analysis will help to provide information on which to devise such guidelines. A similar disparity between guidelines was shown in an international survey of 108 neonatal units published a decade ago. ${ }^{16}$ The lack of progress in this area has been hampered by the lack of an evidence base; but given the rarity of kernicterus hard evidence is not likely to be forthcoming. In our view, if nationally agreed guidelines were adopted and a register of cases of kernicterus maintained, at least the neonatal community could move forward because there would be an opportunity to evaluate whether the levels chosen actually prevented cases of kernicterus occurring. Since we commenced this project, NICE has commenced work on a guideline for neonatal jaundice. This response to the call for action by the UK neonatal community has been warmly welcomed.

Our review of the available guidelines for practice in the UK shows that most units prefer charts, and graphical representation does have the advantage of showing the trend and the rate of rise. Our experience in reviewing these charts has shown that the clarity and presentation could be considerably improved if a few basic rules were followed. We have summarised our suggestions as an Appendix to this article, and have produced an Excel spreadsheet which generates a separate chart for each week of gestation, which is also available to download online.

Funding: This work was undertaken at UCLH/UCL, which received a proportion of funding from the Department of Health's NIHR Biomedical Research Centres funding scheme.

Competing interests: JR has provided advice to lawyers advising both claimants and defendants in litigation regarding kernicterus. She was appointed as Chair of the NICE Guideline Development group on neonatal jaundice in 2007, after the work for this article was carried out.

\section{REFERENCES}

1. Jackson JC. Adverse events association with exchange transfusion in healthy and ill newborns. Pediatrics 1997:99:1-6.

2. Sugama $\mathbf{S}$, Soeda $A$, Eto $Y$. Magnetic resonance imaging in three children with kernicterus. Pediatr Neurol 2001;25:328-31.

3. Okumura A, Hayakawa F, Kato T, et al. Preterm infants with athetoid cerebral palsy: kernicterus? Arch Dis Child 2001;84:F136-7.

4. Bertini G, Dani C, Fonda C, et al. Bronze baby syndrome and the risk of kernicterus. Acta Paediatr 2005;94:968-79.

5. American Academy of Pediatrics. Management of hyperbilirubinemia in the healthy term newborn. Pediatrics 1994:94:558-65.
6. American Academy of Pediatrics. Management of hyperbilirubinemia in the newborn infant 35 or more weeks of gestation. Pediatrics 2004;114:297-316.

7. Maisels MJ, Watchko JF. Treatment of jaundice in low birthweight infants. Arch Dis Child 2003;88:459-63.

8. Watchko JF, Maisels MJ. Jaundice in low birthweight infants: pathobiology and outcome. Arch Dis Child 2003;88:455-8.

9. Ip S, Cheung M, Kulig J, et al. An evidence-based review of important issues concerning neonatal hyperbilirubinaemia. Pediatrics 2004;114:e130-53.

10. Bhutani VK, Johnson L, Sivieri EM. Predictive ability of a predischarge hour-specific term newborns. Pediatrics 1999:103:6-14.

11. Finlay HVL, Tucker SM. Neonatal plasma bilirubin chart. Arch Dis Child 1978;53:90-1.

12. Dodd KL. Neonatal jaundice. Arch Dis Child 1993;68:529-31.

13. Cockington RA. A guide to phototherapy in the management of neonatal hyperbilirubinaemia. J Pediatr 1979;95:285-7.

14. Knupfer M, Pulzer F, Gebauer C, et al. Predictive value of umbilical cord blood bilirubin for postnatal hyperbilirubinaemia. Acta Paediatr 2005:94:581-7.

15. Maisels MJ, Kring E. Transcutaneous bilirubin levels in the first 96 hours in a normal newborn population of $>35$ weeks' gestation. Pediatrics 2006:118:1169-82.

16. Hansen TWR. Therapeutic approaches to neonatal jaundice: an international survey. Clin Pediatr 1996:309-16.

17. Ives NK. Neonatal jaundice. In: Rennie JM, ed. Roberton's textbook of neonatology 4th edn. Philadelphia: Elsevier, 2005: 661-78.

18. Skae MS, Moise J, Clarke P. Is current management of neonatal jaundice evidence based? Arch Dis Child 2005:90:540.

19. Bhutani VK, Gourley GR, Adler S, et al. Noninvasive management of total serum blirubin in a multiracial predischarge newborn population to assess the risk of severe hyperbilirubinemia. Pediatrics 2000;106:1-9.

\section{APPENDIX}

Having reviewed the range of UK practice we propose that (while awaiting guidance from NICE) the threshold for an exchange transfusion should be placed at a level of total serum bilirubin in $\mu \mathrm{mol} / \mathrm{l}$ equivalent to 10 times the gestational age (in weeks) of the baby at birth, after $72 \mathrm{~h}$ of age. Phototherapy should be started at a level $100 \mu \mathrm{mol} / \mathrm{l}$ below that value. A guideline for the first $72 \mathrm{~h}$ of life can be created by joining the exchange plateau to the y axis at a point equivalent to $40 \mu \mathrm{mol} / \mathrm{l}$ at birth. We suggest that the line generated by this process be used as a trigger for monitoring bilirubin levels regularly in the first $24 \mathrm{~h}$ rather than initiating phototherapy; our consensus review showed that few would regard a level of $40-80 \mu \mathrm{mol} / \mathrm{l}$ as an indication to start phototherapy even in very preterm babies. An Excel spreadsheet which can be used to generate gestation-specific charts is available to download. Once phototherapy has begun, bilirubin levels should be monitored every $6 \mathrm{~h}$ in order to ascertain the rate of rise, which should then be controlled below 5 (certainly below 10) $\mu \mathrm{mol} / / / \mathrm{h}$. It is important to ensure that phototherapy equipment is properly maintained and provides adequate irradiance, and if appropriate equipment is used the bilirubin levels should start to fall. If a rising trend continues, and once a level of bilirubin which is $50 \mu \mathrm{mol} / \mathrm{l}$ below the exchange threshold is reached, then the baby should be carefully reviewed for risk factors which may indicate the need for an exchange transfusion urgently. The frequency of bilirubin measurement should be increased to every $4 \mathrm{~h}$ and every attempt made to optimise phototherapy treatment, with recourse to exchange transfusion if the baby's bilirubin levels are not controlled or the baby exhibits neurological signs. Factors such as a low albumin, ongoing significant acidosis (metabolic or respiratory), septicaemia, previous rate of rise and the presence of any abnormal neurological signs should be taken into account when considering whether to perform an exchange transfusion at this stage, at a bilirubin level $50 \mu \mathrm{mol} / \mathrm{l}$ lower than the guideline threshold. serum bilirubin for subsequent significant hyperbilirubinemia in healthy term and near- 
We suggest that future charts and guidelines should:

- provide guidance for management during the first $72 \mathrm{~h}$ of life when bilirubin levels are still rising, stressing the importance of lower levels for intervention and monitoring the rate of rise at this time;

- make decisions on total bilirubin levels unless the conjugated component is more than $50 \%$ of the total;

- start the plateau at $72 \mathrm{~h}$, based on the work of Bhutani and others regarding the pattern of bilirubin rise in normal term newborns ${ }^{19}$,

- make sensible choices regarding the bilirubin level at birth and hence the origin of the chart; the cord blood bilirubin level is not 0 , nor is it normally as high as $150 \mu \mathrm{mol} / \mathrm{l}$ (unconjugated bilirubin crosses the placenta and is metabolised and excreted by the mother). We suggest using a level of $40 \mu \mathrm{mol} / \mathrm{l}$ to represent the cord bilirubin level at which further monitoring is indicated;

- choose a "phototherapy line" after $72 \mathrm{~h}$ which is in line with recommendations which exist, and which distinguishes between gestation groups (ie, does not lump together all preterm gestations below 37 weeks). We propose a level $100 \mu \mathrm{mol} / /$ below 10 times the gestational age of the baby, in weeks;

- maintain a reasonable gap in bilirubin levels between phototherapy and exchange guidelines in order to reflect the fact that obtaining blood suitable for an exchange, and inserting vascular catheters, takes several hours, and we propose a gap of $100 \mu \mathrm{mol} / /$ because phototherapy is safe and this allows the opportunity to review the situation if a level $50 \mu \mathrm{mol} / \mathrm{l}$ below the exchange threshold is reached;

- specify sickness criteria for exchange, but not for phototherapy, because the riskbalance analysis is in favour of a low "catch-all" level for the use of phototherapy. Defining illness is difficult and makes such guidelines harder to implement. We suggest careful review of babies whose bilirubin level continues to rise when phototherapy is started and reaches $50 \mu \mathrm{mol} / \mathrm{l}$ below the exchange threshold, with consideration of whether there are factors present at the time which might be influencing the entry of free bilirubin into the brain, such as acidosis, septicaemia, drug treatment or low albumin;

- provide guidance about the frequency of monitoring of bilirubin levels during phototherapy, and include indications for stopping treatment. We suggest 6-hourly monitoring as the standard, with advice to stop phototherapy when the level of bilirubin has fallen to a level $50 \mu \mathrm{mol} / \mathrm{l}$ below the threshold for treatment. Consideration should be given to checking this using two successive measurements to allow for the inaccuracy of near-patient testing. A check for rebound should be carried out around $8 \mathrm{~h}$ after stopping treatment.

\section{Images in neonatal medicine}

\section{Congenital lumbar hernia}

Lumbar hernias are very rare, ${ }^{1}$ and congenital lumbar hernia is rarer still although no reliable figures have been published. We report such a case.

The baby girl delivered by spontaneous vaginal delivery at term with a normal birth weight. All her antenatal ultrasound scans had been reported as normal including the last scan performed about a week before delivery.

The baby breast fed well and passed urine and meconium normally. On examination, there was a soft fluctuant, nontender swelling on her right iliolumbar area measuring $4 \mathrm{~cm}$ $\times 4 \mathrm{~cm}$. It was not attached to the skin or subcutaneous tissue and did not trans-illuminate. It was reducible but increased in size when she cried or was distressed. Bowel sounds were heard over the swelling. Ultrasound scan revealed a right-sided congenital lumbar hernia which was referred to the paediatric surgeons and scheduled for repair.

\section{O Omolokun, C Woolley, R Evans}

\section{Competing interests: None.}

Patient consent: Parental consent obtained.

Accepted 20 April 2009

Arch Dis Child Fetal Neonatal Ed 2009;94:F327. doi:10.1136/adc.2008.151753

\section{REFERENCE}

1. Gupta H, et al. Congenital lumbar hernia. Indian Pediatrics 2004:41:853.
Picture taken by Medical illustration system, Cwn Taf NHS Trust with permission from parent for teaching and research.
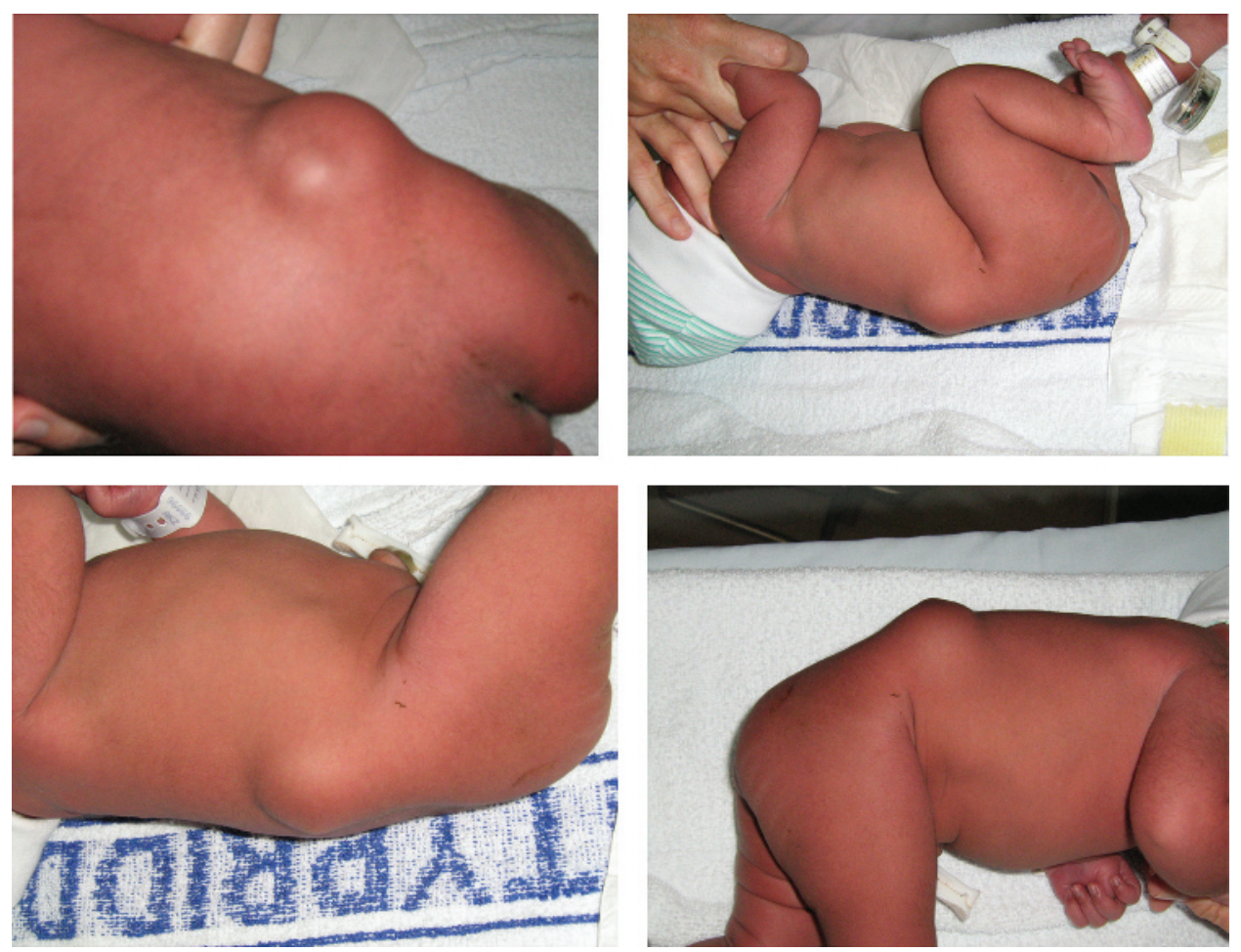